\title{
Vertica-ML: Distributed Machine Learning in Vertica Database
}

\author{
Arash Fard \\ Vertica \\ arash.jalal-zadeh- \\ fard@microfocus.com
}

\author{
Anh Le \\ Vertica \\ anh.tri.le@microfocus.com
}

\author{
George Larionov \\ Vertica \\ george.larionov@microfocus.com
}

\author{
Waqas Dhillon \\ Vertica \\ waqas.dhillon@microfocus.com
}

\begin{abstract}
A growing number of companies rely on machine learning as a key element for gaining a competitive edge from their collected Big Data. An in-database machine learning system can provide many advantages in this scenario, e.g., eliminating the overhead of data transfer, avoiding the maintenance costs of a separate analytical system, and addressing data security and provenance concerns. In this paper, we present our distributed machine learning subsystem within the Vertica database. This subsystem, Vertica-ML, includes machine learning functionalities with SQL API which cover a complete data science workflow as well as model management. We treat machine learning models in Vertica as first-class database objects like tables and views; therefore, they enjoy a similar mechanism for archiving and managing. We explain the architecture of the subsystem, and present a set of experiments to evaluate the performance of the machine learning algorithms implemented on top of it.
\end{abstract}

\section{CCS CONCEPTS}

- Information systems $\rightarrow$ Data analytics; Data warehouses; Clustering; • Computing methodologies $\rightarrow$ Distributed algorithms; Machine learning.

\footnotetext{
Permission to make digital or hard copies of all or part of this work for personal or classroom use is granted without fee provided that copies are not made or distributed for profit or commercial advantage and that copies bear this notice and the full citation on the first page. Copyrights for components of this work owned by others than the author(s) must be honored. Abstracting with credit is permitted. To copy otherwise, or republish, to post on servers or to redistribute to lists, requires prior specific permission and/or a fee. Request permissions from permissions@acm.org. SIGMOD'20, June 14-19, 2020, Portland, OR, USA

(C) 2020 Copyright held by the owner/author(s). Publication rights licensed to ACM.

ACM ISBN 978-1-4503-6735-6/20/06 ..\$15.00

https://doi.org/10.1145/3318464.3386137
}

\author{
Chuck Bear \\ Vertica
}

charles.bear@microfocus.com

\section{KEYWORDS}

Database; Machine learning; Big Data; Distributed Computing

ACM Reference Format:

Arash Fard, Anh Le, George Larionov, Waqas Dhillon, and Chuck Bear. 2020. Vertica-ML: Distributed Machine Learning in Vertica Database. In Proceedings of the 2020 ACM SIGMOD International Conference on Management of Data (SIGMOD'20), June 14-19, 2020, Portland, OR, USA. ACM, New York, NY, USA, 14 pages. https://doi. org $/ 10.1145 / 3318464.3386137$

\section{INTRODUCTION}

Advances in computing technology have made machine learning (ML) ubiquitous in all high-value data-driven applications. Indeed, companies in a very wide range of industries, like marketing, finance, healthcare, telecommunication, and security, have realized that applying ML techniques on their collected data can provide them a significant competitive edge. Traditionally, database management systems (DBMSs) have been the core of any data-driven application; therefore, it is natural to think about improving these systems to better support the new data-driven ML-based applications.

There has been a long-lasting discussion about the intersection of ML and database fields, and the ways they can enrich each other [17, 24]. Particularly, there are many motivations for pushing ML and statistical methods into DBMS [12]; e.g., eliminating big volumes of data transfer, avoiding the maintenance overhead of a separate analytical system, and addressing the concerns of data security and provenance. In [2], Agrawal et al. have also presented their vision of how ML and database systems will evolve coming together in order to support Enterprise Grade Machine Learning.

There have been ambitious projects during recent years to tackle this goal. There are libraries like MADlib $[10,16]$ developed to provide a suite of SQL-based ML algorithms within a database engine. These libraries intend to be a layer on top of any DBMS which supports extensible SQL, and 
therefore to be agnostic to the implementation of the underlying system. However, the intrinsic differences of DBMSs has been a barrier. Hence, MADlib is currently supported only for PostgreSQL [23] and Greenplum [8] database systems.

There are also great efforts by several database vendors to utilize statistical learning packages in $\mathrm{R}$ and Python for indatabase ML; for example, Oracle's ORE [20] and Microsoft's SQL MLS [19]. This approach can provide quick access to a rich set of ML libraries, but it will not be able to match the scalability and performance of natively implemented in-database ML algorithms.

There are also a few distributed platforms for applying ML on Big Data. The most popular ones are Spark-MLlib [18] and $\mathrm{H} 2 \mathrm{O}$ [9]. Despite advances in these platforms, they are compute engines, not full-fledged DBMSs. Therefore, while they can play important roles in an ML ecosystem, they do not provide the advantages of an in-database ML system.

In addition to running in-database ML algorithms, the DBMSs can be utilized for managing ML workflow and models [22]. Models are the most important entities generated in an ML application. In an ordinary ML workflow, data scientists may train hundreds of models for a use case and keep only one to be used in production. The model then should be updated periodically or under some conditions to incorporate any changes in the input data. The same way that DBMSs are used to manage the states of an application, a model management system is necessary to ensure effective deployment of ML in production. Different aspects of model management including model governance are still active research topics [26, 28].

The Vertica Analytic Database is a distributed, massively parallel RDBMS that fully complies with ACID requirements and supports SQL standard language [14, 27]. In Vertica, we strive to help our customers to derive the best value from their data. Therefore, we implemented Vertica-ML inside the database to fulfill market demand. We have followed three broad goals in our design.

(1) Vertica as a platform for high performance ML: We aim to empower Vertica users to run high performant ML algorithms inside the database. These algorithms are added based on our customers' demand and market trends. Indeed, there are customers who have used our ML tools from their first version.

(2) A flexible system with many integration options: We know that it is infeasible to implement everything inside Vertica; therefore, we try to make Vertica-ML more convenient and efficient to be used in a bigger ML ecosystem.

(3) Vertica as a model storage and management system: Being aware of the importance of model lifecycle management, we have implemented the basic tools for model management. Eventually, we would like to archive and manage even ML models generated in other platforms.

There have been technical challenges in pursuing these goals. We designed our ML subsystem to take advantage of Vertica's available internal and external building blocks for analyzing Big Data at scale. Nevertheless, we had to devise lacking tools and mechanisms required for ML algorithms. For example, an ML algorithm needs to iterate over the input data until a convergence criteria is satisfied, but Vertica does not support iterative queries in general. Therefore, we sought workarounds to make a single ML query iterative. We also realized that there is an intrinsic overhead in pulling data through Verica's pipeline in each iteration. Therefore, we developed an internal distributed cache which complies with Vertica's resource management system. Efficiently archiving and managing ML models in our distributed platform was another interesting challenge. To address this, we decided to not limit ML models to tabular data structures.

In this paper, we present the architecture of Vertica-ML, our highly scalable ML subsystem. We also demonstrate the experimental results showing the competitive performance of its distributed ML algorithms. Outstanding characteristics of Vertica-ML include the following:

- An intuitive SQL interface for machine learning It treats models as first-class database objects, and allows business analysts and other SQL users to do more advanced data analysis using the same language they are comfortable with.

\section{- A variety of ML algorithms and tools}

In addition to distributed algorithms for training predictive models, the set of SQL functions introduced by Vertica-ML covers different stages of a data scientist's typical workflow.

- A special model object and model management In contrast to previous in-database ML systems which have been oblivious of model management, Vertica-ML provides capabilities to facilitate it.

\section{- Distributed in-memory storage}

It is integrated into the distributed architecture of Vertica DBMS with the ability to spill to disk when needed.

The structure of the paper is as follows. Section 2 briefly introduces the Vertica ecosystem. In section 3, we list the currently supported algorithms, and explain the SQL user interface of our ML functions and the available model management capabilities. Next, Section 4 presents in detail the technical design of Vertica's machine learning subsystem, starting with an overview of Vertica's architecture for model training, and continues with the contributions necessary to support highly performant and scalable in-database ML. We 
also briefly explain the architecture of our model object. Following this, in section 5 experimental results are presented that demonstrate the performance of Vertica ML algorithms compared with those in Spark-MLlib. In section 6, other related works are reviewed. Lastly, section 7 concludes the work.

\section{VERTICA ECOSYSTEM}

Vertica is a true column-store database that is highly optimized for high performance OLAP queries on exabytes of data across hundreds of nodes in production clusters. Vertica has a pure peer-to-peer cluster architecture. That is, any node can be used to launch a query (initiator node), and up to all nodes collaborate on execution (executor nodes).

There have been over one thousand production deployments of Vertica during the last decade, while its core engine has improved constantly to process Big Data faster and in more convenient ways. Nevertheless, there is an emerging need for more complicated analysis of data beyond what is defined in the SQL standard. Particularly, customers are looking to apply machine learning algorithms on their Big Data to achieve more accurate insights and perform predictive analytics. The first step to satisfy this demand was providing an SDK in order to empower the customers to develop their own User Defined Functions (UDF) and run them directly inside the database. Nevertheless, not all users have enough expertise to develop complicated parallel algorithms, nor is it feasible for most companies to dedicate resources to do so. Hence, a wide range of built-in advanced analytical functions like time series, pattern matching, and geospatial analytics are added into the Vertica database. Particularly, we have added an easy-to-use in-database ML toolset into Vertica. The added functions cover different stages of a data scientist's typical workflow including data exploration, data preparation, model training, model evaluation, prediction, model archiving, and model import/export. The ML functions employ Vertica's powerful distributed execution engine to provide highly performant and scalable ML functionality for big data problems. Moreover, the SQL API of these functions and their tight integration with other database functionalities empower users to gain more value from their data in the same system with the same language.

Vertica also provides integration with third-party products like Apache Kafka, Apache Hadoop, and Apache Spark to satisfy the requirements of complex use cases in a variety of scenarios. Figure 1 displays an overview of the Vertica ecosystem for advanced analytics. Vertica supports an easy-to-use integration with Kafka as a high-performance and low-latency mechanism for streaming high volumes of data both to and from third-party message buses. The primary component of interest in the Hadoop integration is
HDFS which its usage as data-lake has become quite popular. Vertica queries can process HDFS data in-place using a mechanism called external table. Likewise, Spark integration provides a connector for very fast data transfer between Vertica and Spark [15].

Similar to the other Vertica functions, the in-database ML functions can take advantage of the rich Vertica ecosystem to support the daily needs of data scientists and business analysts. These functions can also be wrapped in any other language in a client program. $R$ and Python are two favorite languages of data scientists. Hence, we have provided libraries in these two languages to help users harness the power of Vertica in their favorite languages. vertica.dplyr is an R package that provides Vertica backend support for the dplyr package [30]. Users can use vertica.dplyr to perform operations on Vertica tables similar to those for R DataFrames without moving the data into R. The Vertica-ML-Python library mainly wraps the Vertica analytical functions in Python to make them easy to use along with other available functions in Python. These two libraries are open source and available for download from Github ${ }^{1}$.

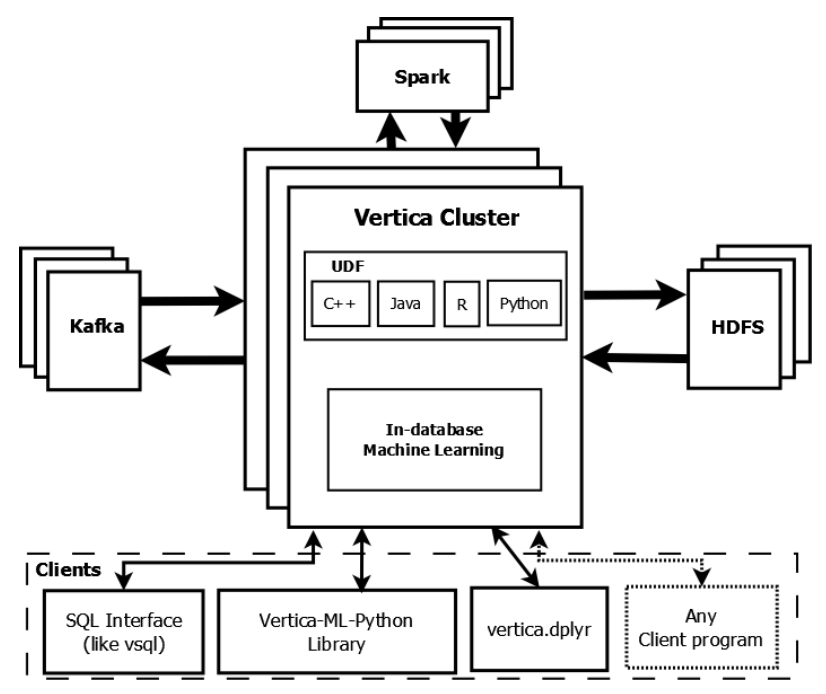

Figure 1: Overview of the Vertica ecosystem

Our focus in this paper is the built-in ML functionality within Vertica. Like the rest of Vertica core, this add-on is implemented in $\mathrm{C}++$. We have used two building blocks inside Vertica for developing ML functions: UDF (User Defined Function) and Metafunction. Because of their important role in the architecture of our ML subsystem, we briefly explain them below.

- UDF: Vertica's SDK [29] enables users to develop their own User Defined Functions and run them inside the

$\overline{{ }^{1} \text { https://github.com/vertica }}$ 
database. These functions are contained in external shared libraries that are developed in $\mathrm{C}++$, Python, Java, or R using the SDK. A UDF can be used anywhere an internal function can be used. Each UDF performs one pass over its input data, and will be executed by the execution engine of Vertica in a distributed fashion to process different segments of data concurrently. Indeed, all of the complicated aspects of developing a distributed piece of analytic code are handled by Vertica. The code of a UDF is just supposed to define how to read a given segment of data, process it, and then write the result out using SDK APIs. The database optimizer will decide at the query planning time how many processes/threads on which nodes are supposed to run the task of the UDF, and how the stream of data segments will be fed to them. This decision mainly depends on the data layout of the input relation, available resources, and defined policies in the system resource manager.

There are also a few UDF types. For development of indatabase ML functions, we mainly use Scalar function (UDSF) where each input row generates an output row with a single value (one-to-one relation), and Transform function (UDTF) where a group of input rows can contribute to several output rows each with multiple values (many-to-many relation). The demonstrated Type I in Figure 2 gives an example of a SQL statement containing a UDF.

- Metafunction: An internal feature for Vertica engineers to develop new SQL functions. As an analogy, the piece of code inside a Metafunction is like $\mathrm{C}++$ client code which connects to the database to run a set of SQL statements and analyze their results. In contrast, the code of a Metafunction does not need a network connection or authentication for running SQL statements because it is already inside the database. Although many of these internal SQL statements will run with the same privileges as the user who has called the Metafunction, the developer can still decide to run some internal SQL statements with superuser privilege when it is safe and suitable. Moreover, all the server's internals like catalog objects are accessible inside a Metafunction. Type II in Figure 2 displays an example of calling a Metafunction.

The main difference between UDFs and Metafunctions is that a UDF can be called as a part of a SQL statement, and it receives one pass of its input data. In contrast, a Metafunction can not be called along with anything else after the SELECT keyword, not even with a FROM clause. A Metafunction contains a procedure which can run any SQL statements, e.g., those that incorporate UDF calls, on any relation to achieve

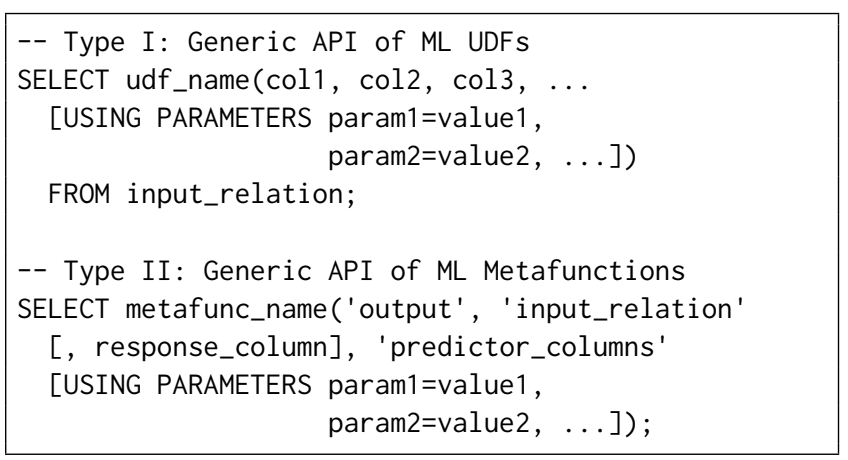

Figure 2: Generic API of ML functions

a goal. The resources allocated to a UDF will be determined by the optimizer and resource management system, but this is not the case for a Metafunction. Therefore, a Metafunction is supposed to be a thin procedure which mainly controls the logic and flow of a complicated task. The usage of UDF and Metafunction in developing our in-database ML will be explained in the next sections.

\section{EXTENSIONS TO SQL}

One of the primary goals of Vertica's ML functionality is to provide an interface that is usable for existing Vertica customers who are comfortable with SQL, and also expressive enough that it can be used by data scientists who code in $\mathrm{R}$ or Python. Vertica seeks to support scalable native solutions for the entire ML workflow. Table 1 summarizes the key ML functionalities currently provided by Vertica. We would like to emphasize that this list grows in every release, and that we have listed here merely what is available at the time of writing this article.

The unique character of our SQL API for in-database ML is that we have defined a specific model object for storing the results of trainings instead of returning them in a tabular format. Relaxing this restriction makes the model structures more amenable to different algorithms and more efficient in practice. For example, a random forest model can contain many decision trees with different sizes where we efficiently save each tree as a binary file. Having a special entity to represent models also allows us to build new tools for model management. The APIs for training and managing ML models are described in this section, and their technical details are explained in section 4 .

\subsection{API of machine learning functions}

From an implementation perspective, our ML functions can be categorized into two groups. Those that need several iterations over the input data are exposed as Metafunctions 
Table 1: Currently available ML functionalities

\begin{tabular}{|l|l|}
\hline Category & Functionality \\
\hline \hline Data Exploration and Preparation & $\begin{array}{l}\text { Statical summary of numerical and categorical columns, Correlation Matrix, } \\
\text { Normalize, One-hot Encoder, Balance, Detect Outliers, Impute, SVD, PCA }\end{array}$ \\
\hline Model Training for Classification & \{Logistic, Lasso, Rigid, Elastic Net\} Regression, Naive Bayes, Random Forest, SVM \\
\hline Model Training for Regression & \{Linear, Lasso, Rigid, Elastic Net\} Regression, Random Forest, SVM \\
\hline Model Training for Clustering & Kmeans, Bisecting-Kmeans \\
\hline Model Evaluation \& Grid-Search & $\begin{array}{l}\text { Feature Importance, Confusion Matrix, Error Rate, ROC, AUC, PRC, F-score, } \\
\text { Lift-Table, R-Squared, MSE, Cross Validation }\end{array}$ \\
\hline Model Management & $\begin{array}{l}\text { Reading the content of a trained model, Import/Export Model, } \\
\text { Altering schema or owner of a model, Dropping a model }\end{array}$ \\
\hline
\end{tabular}

and the others as UDFs. The generic SQL API of both types are displayed in Figure 2.

Most data preparation functions and all model training functions are of type II. For data preparation functions like IMPUTE, the 'output' would be a new view or table. In comparison, for all model training functions the 'output' would be a new model object. The 'input_relation' can be a view, a table, or an external table that contains the input data for training. The 'response_column' for supervisedlearning algorithms is the name of a column in the input relation that contains the response values, also known as targets. Similarly, the 'predictor_columns' are the names of the columns containing the predictor values, also known as features. Each function may have several optional parameters that are listed with their assigned values after the USING PARAMETERS keyword.

The data exploration, prediction, and model evaluation functions are of type I. The input relation for these functions can be specified using a FROM clause where its columns are the inputs of the function. Parameter assignment is similar to type II. Nevertheless, some of the parameters might be mandatory depending on the task of the function; for example, model_name is a necessary parameter for predict functions.

Figure 3 demonstrates an example use case of the ML functions. Here, we use the Diamonds-Kaggle dataset [13] and try to estimate the price of a diamond based on its characteristics. For the sake of space, we assume that the data is already split randomly into two train and test tables. The SUMMARIZE_NUMCOL function is used for exploring the data distribution of the numerical columns in the training data. Then, the NORMALIZE_FIT function normalizes these columns using the robust zscore method. It creates a 'normalize_model', and returns a normalized view of the train data as well. A random-forest model is trained on this view via the RF_REGRESSOR function. In order to apply the model on the test data and measure its quality of the prediction, a normalized view of the test data is created first using the APPLY_NORMALIZE function. Eventually, the PREDICT_RF_REGRESSOR and MSE functions are called for generating predicted values and evaluating their overall quality respectively. You can find a complete version of this example in our blog-post ${ }^{2}$.

It is also worth mentioning that views in Vertica are not materialized. They can be used instead of tables in the intermediate steps for pipelining operations and forcing lazy evaluation.

\subsection{API of Model Management}

When a Vertica ML function trains a model, it will be automatically archived in the database as a special catalog object associated with a schema (public schema by default). Models enjoy the same management capabilities as the other catalog objects. The list of all archived models along with their metadata; e.g., name, schema, owner, type, category, size, and creation time, is available in a system table named models. The type of a model reflects the ML algorithm that has trained it. In comparison, its category represents the ML system used for training the model. Currently, the only available category is VERTICA_MODELS, but it will be extended in the future to have new categories like PMML when we add support for importing models from other ML systems. Model metadata can be used for model governance; i.e., to find out when a model was created and who owns it.

The trained models can be used via several provided apply and predict functions for data transformation or scoring. Moreover, there are two generic functions for reading the contents of any model. The get_model_summary function returns a text summary of the content of a model. The get_model_attribute function can return different attributes stored in a model in tabular format, e.g., the set of

\footnotetext{
${ }^{2}$ https://www.vertica.com/blog/verticas-in-database-random-foresthighly-scalable-and-easy-to-use
} 


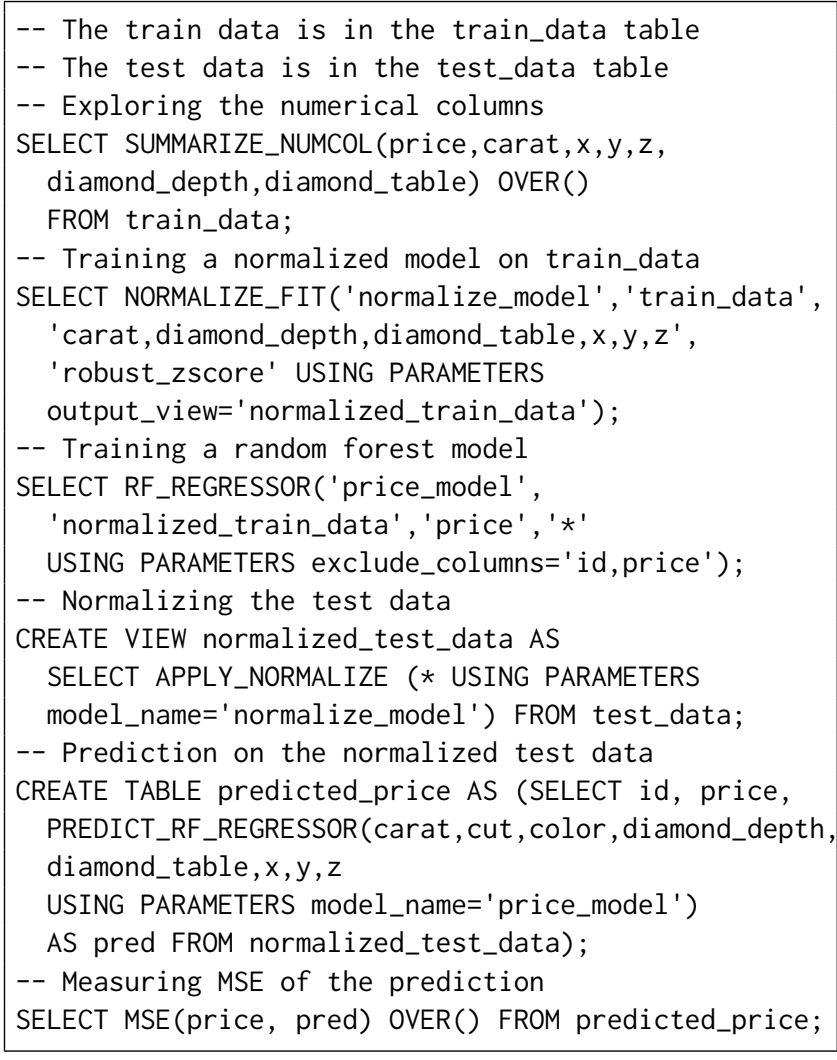

Figure 3: An example of calling ML functions

coefficients of a logistic regression model. These two functions are very useful not only for monitoring models, but also for managing them. The summary of a model contains information like the relation name (input table), the columns in that relation, and the tuning parameters used at the time of training. This information can be used to reproduce a similar model. It is also possible to search among the models over their trained attributes using the returned results of the get_model_attribute function.

Similar to tables and views, the ALTER and DROP statements can be used in order to rename, change the owner, or delete a model. The initial owner of a model is the user who trained the model, and it is the only user who has the usage and management privileges of the model by default. Nevertheless, the owner of a model can permit other users to use it as well using the GRANT statement.

Users can also export the archived models as binary files and import them into another Vertica cluster with the same or higher version.

\section{VERTICA-ML ARCHITECTURE}

The Vertica database is designed for data warehouses and other Big Data workload applications where speed and scalability are crucial requirements. Nevertheless, in order to build efficient and scalable functions on top of this platform for training ML models, we had to extend its architecture.

\subsection{Distributed ML algorithm}

At a high level of abstraction, a machine learning model $M$ is a parametric data model which is trained on a given set of data points $X=\left\{x_{1}, x_{2}, \ldots, x_{n}\right\}$ in order to minimize a cost function:

$$
\operatorname{Cost}(M, X)=\sum_{i=1}^{n} C\left(x_{i} \mid M\right)
$$

where $C$ is the cost function per data point.

The number of parameters in a model, $M\left(\mu_{1}, \mu_{2}, \ldots, \mu_{d}\right)$, depends on the training algorithm and the dimension of the training data points. The training algorithms usually need to iterate over the training data several times until the value of cost decreases below a threshold (converges), or the number of iterations reaches a predetermined maximum number. In each iteration, the model is updated using an update function:

$$
M_{j+1} \leftarrow F\left(M_{j}, X\right)
$$

Tables in Vertica are limited to 1600 columns, but they can contain billions of rows. In this context, each row represents a data point. To design a distributed ML algorithm for Big Data problems, Data Parallelism is a reasonable choice. In this approach, the training data is split into disjoint sets, $X=\cup_{k=1}^{m} S_{k}$, and then local models will be trained in parallel on each split. Algorithm 1 displays some very high level pseudocode of such a distributed ML algorithm. First, an initial model is built (line 2). Then, there is an iteration to optimize the model (line 5). The distributed execution engine performs computation on the local splits of the data in each iteration to calculate the local models and their local cost value (lines 7 to 10). These local models are then aggregated on a single process on the initiator node to update the global model (line 11). The logic of the aggregation function is different for each ML algorithm. Subsequently, the global cost will be calculated as the summation of the local costs (line 12). The training process will iterate until either the global cost is decreased below a threshold or the number of iterations reaches a specified limit. In the following subsections, we explain the architecture of Vertica-ML for implementing such a distributed ML algorithm, and highlight the components added to the Vertica platform for this purpose. 


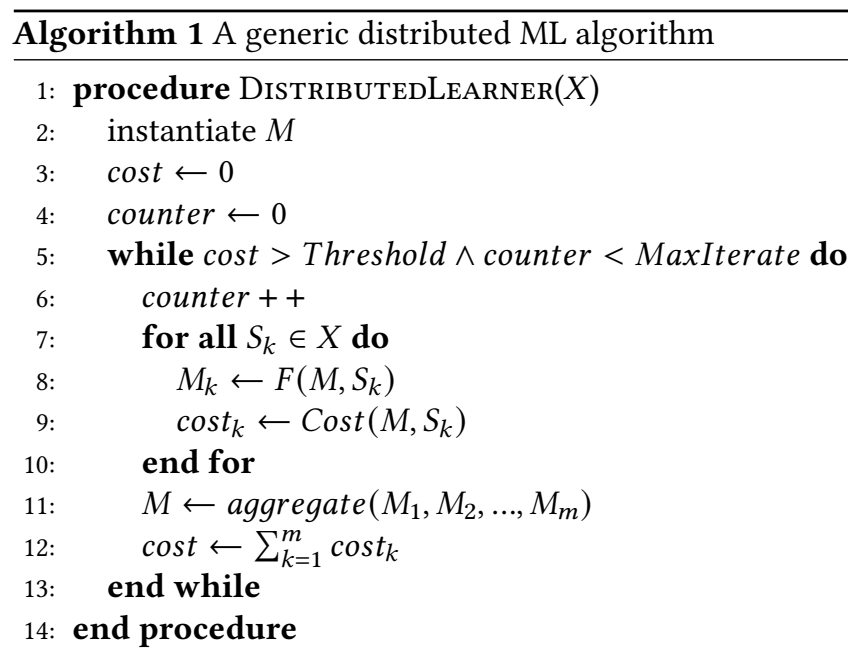

\subsection{Model training}

The overall architectural diagram for a model training function, e.g., logistic regression, is illustrated in Figure 4. Each model training function is composed from a Metafunction and several UDTFs, but only the Metafunction is exposed to users. When it is called, it interprets its input parameters and creates a model object (corresponding to line 2 of Algorithm 1). In short, each model is a catalog object and its trained data is stored in a set of binary files on Vertica's built-in Distributed File System (DFS). We will explain more about them in section 4.5 .

The control logic of the algorithm is also implemented inside the Metafunction. As its first step, it calls a UDTF for caching the content of an input table into a blob. A blob is a distributed buffer we built for our ML framework to keep data in memory temporarily. We will explain the design and structure of blobs in section 4.4 .

Then, a set of UDTFs are called to perform the desired computation on the data from the blob and update the DFS files of the model iteratively. The training iterations are also controlled by the Metafunction (corresponding to lines 5 to 13 of Algorithm 1). The iteration will terminate after either meeting convergence criteria or reaching a specified maximum number of iterations.

\subsection{Parallelism Control for UDTF}

Calling a UDTF in a session spawns several threads in each node of a Vertica cluster to execute the desired function in parallel on different splits of data. In general, the number of threads per node is smartly determined by the query planner based on available resources and settings of the session. We realized that for developing efficient ML algorithms we needed more control over the number of threads, and new

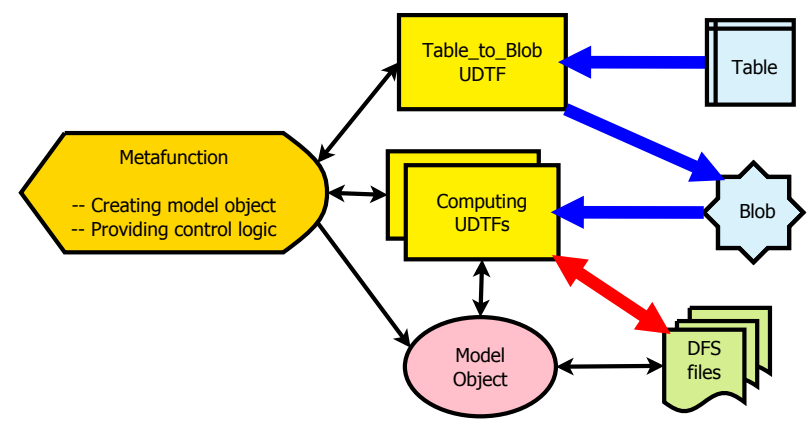

Figure 4: Architectural diagram of model training

ways for multitasking and data sharing among them. Therefore, we added two main extensions to the UDTF framework in order to address these issues:

- We added a new mechanism so that a UDTF can demand for special cluster nodes and a specific number of threads on each node from the optimizer unit at query-planning time.

- To provide task parallelism, we made the UDTF's threads aware of their integer index numbers. Thus, threads on each node can be programmed to perform different tasks based on their own index.

The first extension is used for working with blobs which will be explained in section 4.4. A usage example for the second extension is when all the threads of a computing UDTF need to have access to the data of a model. In this case, only the leader thread, which is identified with index zero, reads the model files from DFS into a buffer and shares its pointer with other threads. This way, there would be no I/O congestion regardless of the number of threads per node.

\subsection{Distributed In-memory Storage}

An important difference between an ML algorithm and a typical SQL query is its need to iterate over the entire input dataset many times. Therefore, minimizing reading time is crucial. Vertica's data layout for tables uses separate files for different columns, and each file is compressed and encoded. Hence, the data needs to be read from files, decompressed, decoded, and constructed in blocks to be consumed by the execution engine. We observed that repeatedly reading the data through the ordinary data pipeline causes a lot of unnecessary overhead per iteration. Also, in many ML algorithms we need to store some temporary values corresponding to each row of the input data and update them in each iteration. We could use a temporary table for this purpose, but again it would be an expensive operation.

To eliminate these overheads, we designed a distributed in-memory storage called blob. We decided to develop it by ourselves instead of using a third-party library for practical 
reasons: (1) Our requirements for such a distributed data structure were simple, so it could be implemented in a reasonable amount of time. (2) We preferred to avoid the chance of any bugs in a complicated third-party library. (3) It is important to be able to control its memory allocation from the Vertica resource manager unit.

Each ML algorithm may create one or more blobs to cache its input data and store any temporary intermediate data. Data can be stored in any format in a blob; i.e., it does not need to be tabular. Also, when the size of the data grows bigger than the permitted blob size for a session, it will spill to disk transparently. We have used an available mechanism in Vertica, named resource pool, to make the max blob size of a session adjustable.

We developed the blob API as an extension to the C++ UDTF framework. The API smoothly integrates with Vertica's existing mechanism within the optimizer for choosing thread count and data distribution.

Figure 5 displays the creation and usage of a blob in an ML algorithm in more detail. For simplicity, the illustrated cluster has three nodes, and each UDTF has two threads per node. In a normal use case, the rows of a table are evenly segmented among cluster nodes using a hash function. An ML algorithm initially calls the Table_to_Blob UDTF to cache the contents of the input table into a blob. At this stage, we let the database optimizer determine the concurrency level of the UDTF, i.e., the number of threads per node. As this happens at the query-planning time, creation of a blob with the same number of chunks per node can be requested as well. Chunks are the logical building blocks of a blob. This abstraction allows writing to the blob without worrying about synchronization between threads. As illustrated in Figure 5a, a UDTF's threads receive disjoint sets of rows from their corresponding table segments and store them in their assigned blob chunks.

After caching the input data into a blob, any UDTF in the same session can have access to this data. As it is illustrated in Figure 5b, the ML Metafunction calls a nested query containing UDTF1 in the inner statement and UDTF2 in the outer one. In this stage, UDTF1 learns about the number of blob chunks on each node at the query-planning time and demands for proper number of threads so that there would be one thread corresponding to each blob chunk. In comparison, UDTF2 will have only one thread running on the initiator node. Indeed, UDTF1's threads read splits of data and perform the foreach loop of Algorithm 1 (lines 7 to 10) in parallel. UDTF2 receives the results of UDTF1's threads, aggregates them, and updates the model. The nested query will be called iteratively until the iteration condition is satisfied.

Any blob created for an algorithm is cleaned up after the model training is finished. Moreover, the lifetime of a blob is
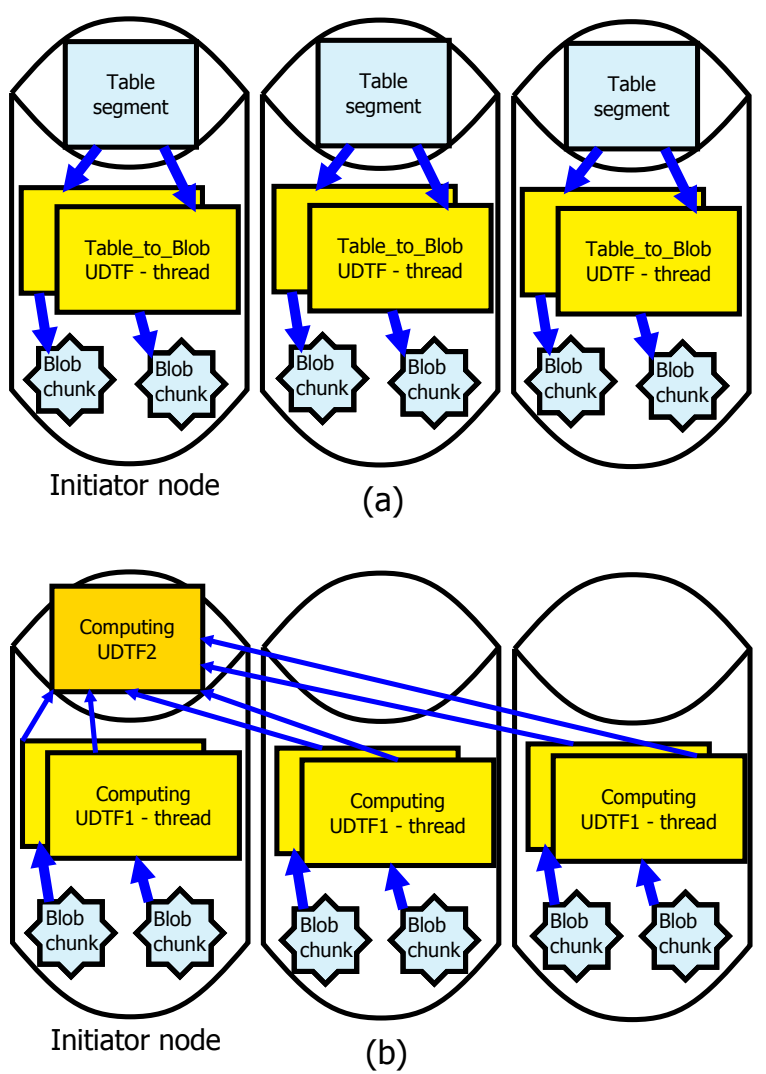

Figure 5: (a) Concurrently caching table segments to blob chunks (b) Parallel processing by threads of UDTF1 on blob chunks and aggregating their results by UDTF2

limited to its session; hence, it will be automatically wiped out if its session ends for any reason.

To measure the impact of blob usage on the running time of an algorithm, we performed an experiment with a dummy function. The function calculates the summation of all elements of an input table in which all the columns are of type float, and repeats it 100 times. The first version of this function is implemented using an ordinary UDTF which reads the column values directly from the input table in each iteration. In contrast, the second version caches the input table into a blob at the beginning, and then in each iteration its UDTF reads the data from the blob. Figure 6 illustrates the impact of the blob usage on the running time of the dummy function. In this experiment, we have used the Million Song dataset. The dataset characteristics and the hardware configuration used in this experiment will be mentioned in section 5 . 


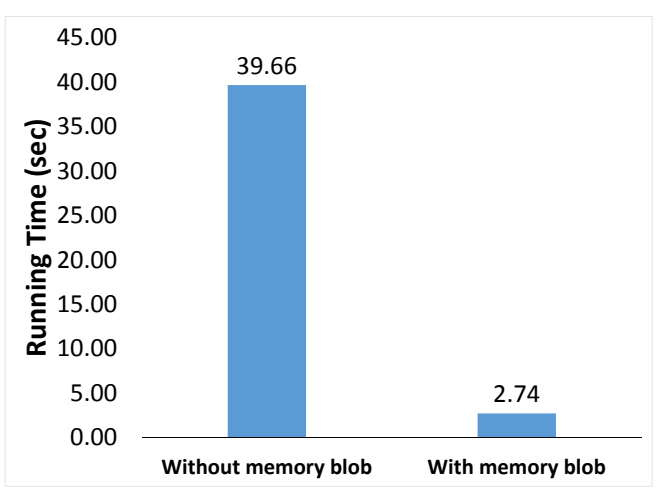

Figure 6: Impact of using blob in a dummy function

\subsection{Model object}

An important advantage of Vertica's in-database ML is its capability to efficiently archive and manage models. Each model in Vertica is represented by a catalog object and benefits from a similar mechanism as that used for access policy and management of a table. For instance, a model must belong to a database schema; it has an owner, and its access can be delegated to other users.

The model catalog object keeps only metadata of a model like its name, owner, type, and creation time. The real data of a model, e.g., trees in a random-forest model, are stored in its associated DFS files. Vertica has a built-in distributed file system for its internal use cases. Storing the model's data on DFS has two main advantages: (1) In the training phase when the model is updated in each iteration by a single process (refer to UDTF2 in Figure 5b), it is replicated on all other Vertica nodes; hence, all the distributed threads (refer to UDTF1 in Figure 5b) will have access to the updated model in the next iteration. (2) At the time of prediction, all threads of the predict function will have access to a local copy of the model on all Vertica nodes.

To create a proper relation between a model and its DFS files, we had to slightly modify the DFS structure. All the folders and DFS files in Vertica have catalog object representatives. Figure 7 displays the dependency tree with respect to DFS objects in Vertica. This tree is a fundamental concept for managing delete and access policies of objects; for example, to remove a schema, all its dependent objects must be removed first. The root of the dependency tree for all objects is the database object. In the old DFS structure, there is a root DFS folder depending on the database, and all other DFS folders and files are its children, as can be observed on the left branch of Figure 7. We modified this structure such that now each model object has its own DFS root folder depending on it. All the DFS folders and files that would be generated under this root during the model training will belong to that particular model.

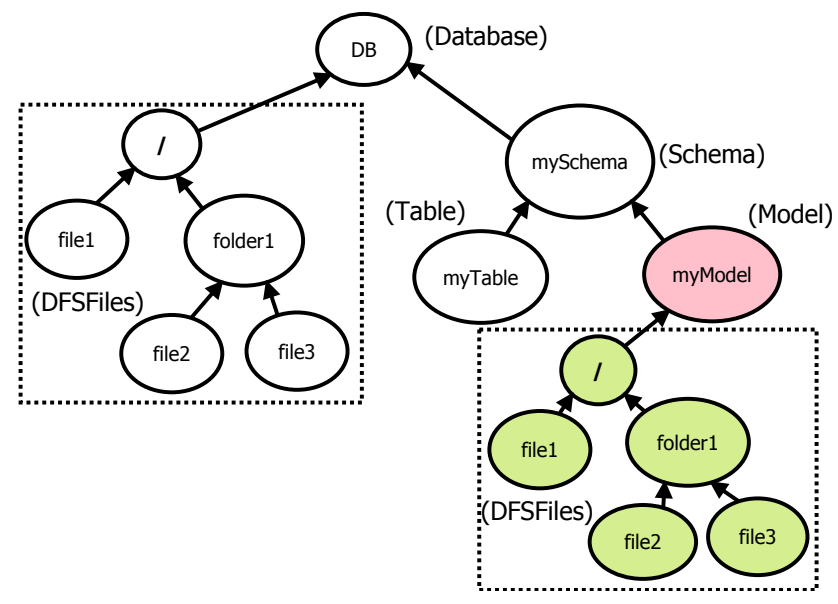

Figure 7: An example of the dependency tree in Vertica

\subsection{Example Algorithm: Kmeans Clustering}

We have thus far described in detail how Vertica-ML is designed for implementing various distributed machine learning algorithms. To provide an example, in this section we explain how we have implemented kmeans clustering, one of the most popular clustering algorithms.

4.6.1 Algorithm. Kmeans is an unsupervised algorithm which accepts datasets with all numerical features, and assigns the data points into $k$ clusters [7]. The dataset can have any number of dimensions and any number of points, as long as a distance metric is provided to measure distance between the points and the centers of the $k$ clusters they are assigned to. The euclidean distance is the most popular metric for this purpose. The algorithm starts with $k$ initial cluster centers and iterates over the data, updating the centers to minimize a cost function. The cost function of kmeans is the withincluster variance, or sum of squares (withinSS), which can be expressed as follows,

$$
\text { withinSS }=\sum_{i=1}^{k} \sum_{x \in C l_{i}}\left\|x-c_{i}\right\|^{2}
$$

where $C l_{i}$ stands for cluster $i$ (out of $k$ ), $x$ stands for one data point vector, and $c_{i}$ stands for the center of the $C l_{i}$ cluster. The goal of the algorithm is to assign points to clusters such that the total within-cluster sum of squares is minimized.

Selecting a proper set of initial centers can impact not only the number of iterations for convergence, but also the final result. The initial $k$ centers may be been given by the 
user, randomly picked from the input data points, or selected using a heuristic approach. All of these options are provided by Vertica kmeans, and it supports scalable kmeans++ [4], a heuristic approach for finding the optimal initial centers. At each iteration of the algorithm, data points are assigned to their closest center (equation 4), and then the centers are updated to the mean of the newly formed clusters (equation 5). The algorithm stops iterating when there are no significant changes in the center values, or when it reaches a given maximum number of iterations.

$$
\begin{gathered}
C l_{i}^{t}=\left\{x \in X:\left\|x-c_{i}^{t}\right\|^{2} \leq\left\|x-c_{j}^{t}\right\|^{2}\right\} \\
\text { where } j \neq i \wedge 1 \leq j \leq k \\
c_{i}^{t+1}=\frac{1}{\left|C l_{i}^{t}\right|} \sum_{x \in C l_{i}^{t}} x
\end{gathered}
$$

Equations 4 and 5 formulate the steps of reassigning data points to the clusters and calculating the new centers, respectively; $t$ refers to the iteration number.

4.6.2 Distributed Implementation. As outlined in section 3.1, each Vertica ML algorithm consists of a Metafunction and a few UDF functions. The Metafunction contains the overall logic of the algorithm and calls the user defined functions (UDFs) iteratively to perform calculations over the data. The pseudocode for the kmeans Metafunction is represented in algorithm 2 .

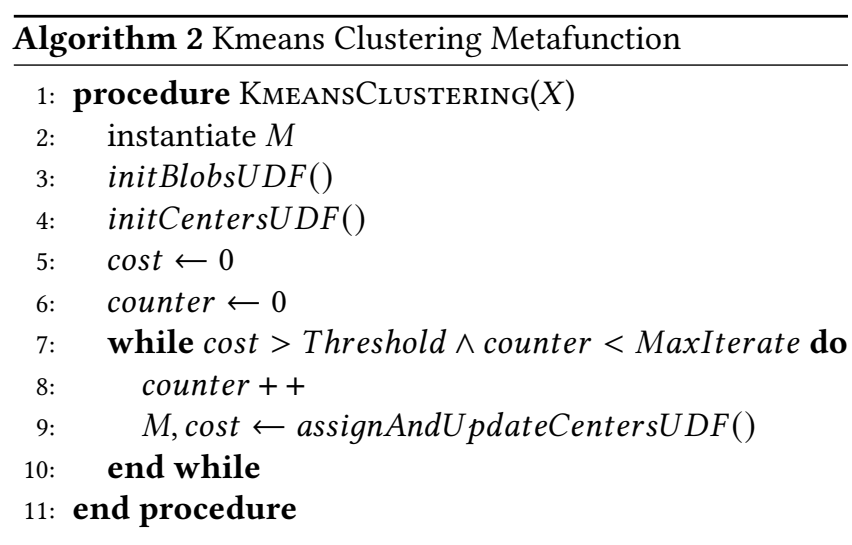

The Metafunction first instantiates a model object $M$ (refer to section 4.5 ) which mainly preserves $k$ centers, each representing a cluster. Then, a UDF (initBlobsUDF) is called to create two blobs, Blob1 and Blob2, with the same distribution layout. Blob1 caches the vectors of the input data and their norm values. The norm values are used for an optimization later during the iterations. Blob2 is used for bookmarking and for each data point it contains the ID of its assigned cluster and the distance from its center. A set of UDFs (initCenters $U D F$ ) are called next to update the model with the initial centers based on the available initialization methods. Lines 7 to 10 of algorithm 2 are a simplified version of the iterative part of the algorithm. The assignAndU pdateCenters $U D F$ function resembles UDTF1 and UDTF2 depicted in Figure 5b. In each iteration of kmeans, threads of UDTF1 read the model and the distributed chunks of Blob1, perform the calculation of equation 4, and then update the corresponding chunks of Blob2 with new cluster IDs and distance values in parallel. At the same time, they calculate their local values for new centers and the cost value related to equation 5, and pass it to UDTF2. UDTF2 aggregates these values to calculate the global value of the cost, and updates the model with the new global centers.

\section{EXPERIMENTAL EVALUATION}

We have conducted several experiments to evaluate the performance of a few implemented in-database ML algorithms. We compare the running times in Vertica for training ML models to similar algorithms in Spark-MLlib. Despite the differences in the implementations, we have made sure that the prediction quality of their outcome models are very close. We use MSE (Mean Squared Error) and AUC (Area Under ROC Curve) to measure the prediction quality of regressors and classifiers respectively.

We have picked Spark-MLlib for comparison because it is a very popular distributed compute engine among data scientists who need to run machine learning algorithms on Big Data. The goal of these experiments is to show that the performance achieved by Vertica-ML is comparable to a popular platform while it provides many additional advantages. Also, we would like to emphasize that it is not straightforward to compare the performance of any two ML training algorithms on these two platforms because the underlying mathematical optimization logic is often quite different, which makes them scale differently when the size of the problem increases in the same dimension. We will show this issue on the running time of the random-forest algorithm with respect to changes in the max depth of its trees.

\subsection{Experimental Setup}

Hardware Configuration. All the experiments were performed on a four-node cluster. Each node has 36 hyperthreaded $1.2 \mathrm{GHz}$ cores (GenuineIntel), 755GB RAM, and is connected to a $10 \mathrm{Gbps}$ network.

Software Configuration. The cluster nodes are running Ubuntu 14.04. Vertica 9.2.0 and Spark 2.4.0 (with Hadoop 2.8.0), which are used in the experiments, have been installed on the same hardware. Nevertheless, we shutdown Spark and Hadoop at the time of Vertica experiments and vice versa. 


\subsection{Datasets}

We have used two real-world datasets in our experiments: Airline dataset [3] and Million Song dataset [6].

Airline dataset: This dataset contains flight arrival and departure details for all commercial flights within the USA, from October 1987 to April 2008. It is a dataset with nearly 120 million records; therefore, it is large enough to measure the scalability of a supervised ML algorithm with respect to the number of records. Using the departure delay of flights in the dataset, we have defined both regression and classification problems. The goal of the regression problem is to predict departure delay of a flight in minutes. The goal of the classification problem is to predict if the departure of a flight would be delayed more than 15 minutes. There are eight predictors in this dataset; two of them are numerical and the rest categorical. We randomly selected $20 \%$ of the data as a test set, then we randomly picked from the remaining data to generate train sets with $10 \mathrm{M}$ (million), 20M, $40 \mathrm{M}$, and $80 \mathrm{M}$ rows. The size of these train sets in Bytes are roughly $0.4 \mathrm{~GB}$, 0.8GB, 1.6GB, and 3.2GB.

Million Song Dataset: This dataset is a freely-available collection of audio features and metadata for contemporary popular music tracks. It contains 90 numerical predictors for each song and its release-date as the response value. Therefore, it is a good dataset for a regression algorithm or any algorithm which requires all predictors to be numerical, e.g., Kmeans and PCA. The goal for the regression problem would be to train a model that predicts the release year of a song from its audio features. The data is already split into train and test sets, with 463,715 and 51,630 records respectively.

\subsection{Performance comparison}

We have performed each experiment five times and reported the average running times. Also, we would like to highlight that we have tuned neither Vertica nor Spark. In general, there aren't many ways of fine-tuning Vertica-ML except for setting concurrency plans and memory caps per query in the resource pools. Since the Vertica database is designed for a concurrent multiuser scenario, these settings specify the max usage of resources for each session such that its workload will have minimal impact on the other concurrent sessions. In comparison, Spark does not support concurrent workloads, but has plenty of other knobs for specifying the resources for running each batch job. For example, the number of partitions of a distributed DataFrame, when it is loaded from a CSV file, can be specified manually; otherwise, Spark will select it automatically based on the size of the data and the available resources.

Figure 8 compares how Vertica and Spark scale as the number of rows increases for training random-forest models on the Airline dataset. Figure $8 \mathrm{a}$ is for the regression function of random-forest, and Figure $8 \mathrm{~b}$ is for the classification function. In these experiments, all the training parameters on the two platforms are the same; the important ones are: max_depth $=5$, ntree $=20$, nbins $=400$.

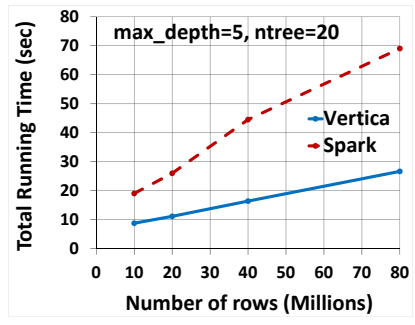

(a) Regression

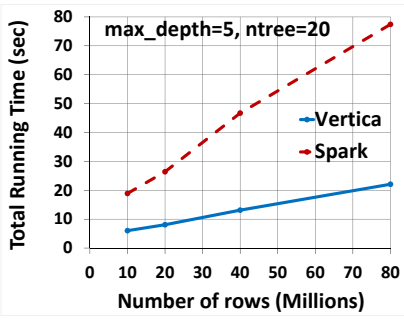

(b) Classification

\section{Figure 8: Random-Forest on Airline dataset}

Figure 9a compares how Vertica and Spark scale as the max depth of trees increases for training random-forest regressor models on the Million Song dataset. For the tests in this chart, the number of trees was fixed to 20 . The prominent difference between the behaviors of Vertica and Spark in Figure 9a is due to the details of their algorithm implementations. The random-forest in Vertica, inspired by the PLANET algorithm [21], starts with Data Parallelism when the training data for growing decision trees is big, and then smartly switches to Model Parallelism when training data on leaf-nodes becomes small. This strategy is to adjust the balance between parallel processing and data communication cost. This transition in the algorithm is the reason behind the sudden change in the behavior of the Vertica random-forest that can be observed in Figure 9a for max_depth bigger than 10 .

Figure 9b compares the scalability of Vertica and Spark with respect to an increase in the number of trees for training random-forest regressor models on the Million Song dataset. For these tests, the max depth of trees was fixed to 15. For both sets of experiments in Figure 9, all algorithm parameters were the same. The one worth mentioning is nbins $=64$.

Figure 10 represents the scalability of the Vertica randomforest algorithm with respect to the number of cluster nodes. Figure 10a displays the speedup factor of random-forest functions on the Airline dataset with $80 \mathrm{M}$ rows for both classification and regression problems on Vertica databases with different numbers of nodes. To measure the speedup factor of a database, we used this equation: speedu $_{i}=T_{1} / T_{i}$, where $T_{i}$ is the running time of the algorithm on the database with $i$ nodes. We can observe that for this dataset the speedup values on four nodes are 3.03 and 2.75 for classification and regression respectively. It is well known that the ratio of communication time to processing time in a parallel algorithm 

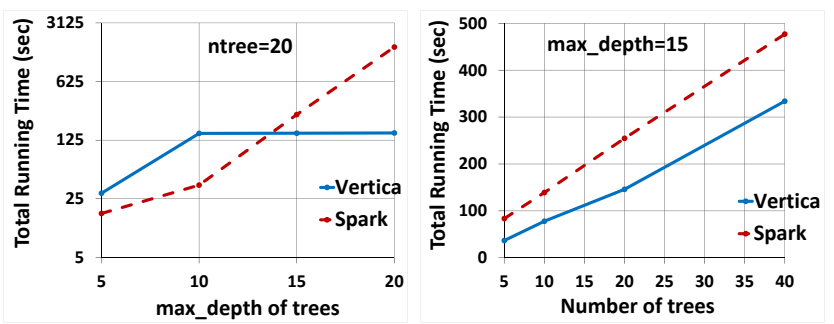

(a) varying trees max_depth

(b) Varying number of trees

\section{Figure 9: Random-Forest on Million Song dataset}

usually decreases with increasing size of input data [25]. This phenomenon is displayed in Figure 10b. To synthesize a dataset with $400 \mathrm{M}$ rows, we copied the Airline dataset of $80 \mathrm{M}$ rows into a new table five times. In this experiment, the speedup values on four nodes have improved to 3.46 and 3.35 for classification and regression respectively.

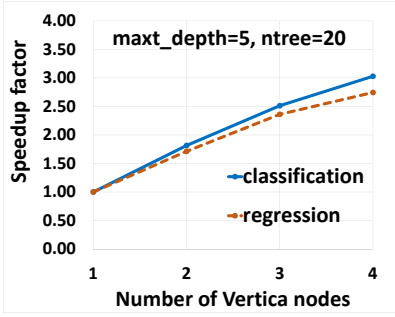

(a) Airline dataset:80M rows

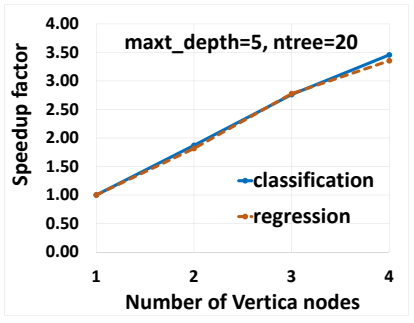

(b) Airline dataset:400M rows

\section{Figure 10: Scalability on the number of nodes}

Among the supervised algorithms that are available in both Vertica-ML and Spark-MLlib, we picked only randomforest to report the comparisons in Figure 8 and Figure 9. We found it much easier to keep the number of iterations the same between the two implementations for this algorithm while their prediction quality is also very similar. In comparison, we realized that the other supervised algorithms like linear and logistic regression converge in different numbers of iterations, unless we force them to run for a relatively small number of iterations which will then cause a difference in the quality of their output models.

We have also compared the running time of three other algorithms, namely kmeans, PCA, and linear-regression on the Million Song dataset. The results can be observed in Figure 11. The selected value of $k$ for kmeans in this experiment is 10 . We also set the max number of iterations for kmeans to 10 and confirmed that none of the implementations converged earlier. In the PCA experiment, the number of components is 5 . Moreover, the optimizer method for linear-regression in both Vertica and Spark in this experiment was the Newton method which made both converge at the first iteration.
It should be noted that a single Vertica training function reads the content of its input table from disk and caches it into a blob, and then iterates over this in-memory data to train the desired model. The procedure in Spark-MLlib is similar but involves calling several functions. At first, we read the input CSV file from HDFS into a DataFrame and cache it in memory. We measure the spent time for this step and name it loading time. Next, we create the desired training object and fit it with the input DataFrame. We name the measured time of this step training time. We report the summation of these two as the total training time for the Spark experiments. As shown in Figure 11, loading time can be a big portion of the total time for Spark. Nevertheless, in this set of experiments Vertica's total running times are still shorter than Spark's corresponding training times.

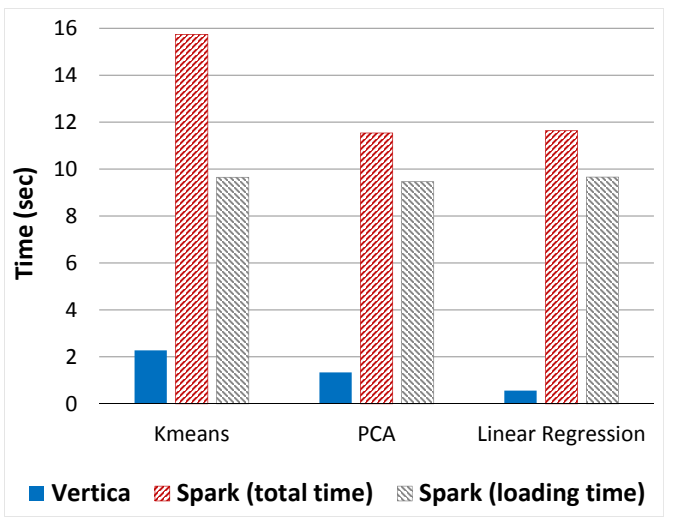

Figure 11: A few algorithms on Million Song dataset

\section{RELATED WORK}

In this section we describe some of the related work in the area of in-database ML and model management.

MADlib [10] is an open source library designed to provide SQL-based ML functions in any relational database that supports extensible SQL. To the best of our knowledge, it is available only on PostgreSQL [23] and Greenplum [8].

Oracle Advanced Analytics [20] is a feature in Oracle database for in-database ML functionality. It is composed of Oracle Data Mining (ODM) and Oracle R Enterprise (ORE). ML functions provided by ODM have SQL APIs and are executed by the database execution engine. The ORE option integrates the $\mathrm{R}$ language with the database so that ML functions and statistical scripts written in $\mathrm{R}$ can be executed by the execution engine of the database. Similarly, Microsoft's SQL Server Machine Learning Services [19] is an add-on to Microsoft's SQL Server for running $\mathrm{R}$ and Python scripts inside the server.

Acknowledging the advantages of in-database ML, in 2018 Google announced the launch of an ML beta library in the 
BigQuery database available on Google cloud [11]. They had linear regression and binary logistic regression algorithms in their initial beta release, and have added multiclass logistic regression and kmeans since then. It is worth mentioning that the first Vertica-ML, GA release in 2016, had linear regression, binary logistic regression, and kmeans algorithms.

In [2], Agrawal et al. have investigated the status of Enterprise Grade Machine Learning (EGML) and its requirements at the present, and what it may look like in 10 years. They have specifically focused on two categories that they believe require DB community attention: 1) taking advantage of database in an ML ecosystem for archiving trained models, in-database scoring, and improving the decision making process; 2) data and model management for an ML workflow, e.g., data discovery, access and versioning, model governance and provenance.

Challenges in managing data lifecycle and ML models in production have been the topic of many other recent studies as well. Polyzotis et al. published a survey about these challenges in [22]. They noticed the importance of data management in large-scale ML systems, and described large-scale ML pipelines. They have also surveyed challenges and techniques related to data management for ML.

The opportunities and challenges in model management are also discussed in [28]. The authors explain the evolving opportunities in model management specifically in these directions: model data pipelines, model interoperability, model testing, model monitoring, and model interpretability and fairness. The paper also introduces MODELDB which is an open source software developed for model management. It is basically a metadata database in order to track model building and record provenance information.

Sridhar et al. have also presented their work on model governance and provenance in [26]. Their proposed solution for collecting model information is based on an agent/server architecture. The agents run on each instance of an ML engine, e.g., a Spark cluster, and send governance metadata to the server. Indeed, the server is a database of ML metadata which is used to provide model governance capabilities including provenance/lineage, reproducibility, audit and compliance, leverage, scale and heterogeneity.

\section{CONCLUSIONS}

In this work we have presented Vertica-ML, the in-database distributed ML extension of Vertica for Big Data Analytics. We introduced the SQL API of the ML functions and model management capabilities. We also explained the extensions added to Vertica like the blob structure which provides distributed in-memory storage.

Vertica-ML was first released with Vertica 7.2 in 2016, and has been constantly improved and expanded since then. In addition to developing new in-database distributed ML algorithms, enhancing model management capabilities and integrating with other ML tools are also on our future roadmap. For example, we are planning to make it possible to import PMML [5] models, trained on other ML platforms, into Vertica and to use them for in-database scoring. It will also be possible to export the native Vertica models as PMML models to be used for scoring on other systems, e.g., on an edge node. TensorFlow [1] model importing is also on our roadmap. This feature will allow users to import previously-trained TensorFlow models, archive and use them for prediction inside Vertica.

\section{ACKNOWLEDGMENTS}

The authors would like to thank Ben Vandiver, the former CTO of Vertica, for his invaluable guidance. We are also grateful to the other colleagues who had precious contributions to the project, especially Matthew Saltz, Pratik Dhandharia, Vincent Xu, Mark Fay, Justin Payan, Winston Li, Renze Wang, Ginger $\mathrm{Ni}$, and Lukai Liu.

\section{REFERENCES}

[1] Martín Abadi, Paul Barham, Jianmin Chen, Zhifeng Chen, Andy Davis, Jeffrey Dean, Matthieu Devin, Sanjay Ghemawat, Geoffrey Irving, Michael Isard, Manjunath Kudlur, Josh Levenberg, Rajat Monga, Sherry Moore, Derek G. Murray, Benoit Steiner, Paul Tucker, Vijay Vasudevan, Pete Warden, Martin Wicke, Yuan Yu, and Xiaoqiang Zheng. 2016. TensorFlow: A System for Large-Scale Machine Learning. In 12th USENIX Symposium on Operating Systems Design and Implementation (OSDI 16). Savannah, GA, 265-283.

[2] Ashvin Agrawal, Rony Chatterjee, Carlo Curino, Avrilia Floratou, Neha Gowdal, Matteo Interlandi, Alekh Jindal, Kostantinos Karanasos, Subru Krishnan, Brian Kroth, et al. 2019. Cloudy with high chance of DBMS: A 10-year prediction for Enterprise-Grade ML. arXiv preprint arXiv:1909.00084 (2019).

[3] ASA. 2009. Airline on-time performance. stat-computing.org/dataexpo/ 2009/

[4] Bahman Bahmani, Benjamin Moseley, Andrea Vattani, Ravi Kumar, and Sergei Vassilvitskii. 2012. Scalable K-means++. Proc. VLDB Endow. 5, 7 (March 2012), 622-633. https://doi.org/10.14778/2180912.2180915

[5] DMG. 2019. PMML website. dmg.org/pmml/v4-4/GeneralStructure. html

[6] Dheeru Dua and Efi Karra Taniskidou. 2017. UCI Machine Learning Repository. http://archive.ics.uci.edu/ml

[7] Jerome Friedman, Trevor Hastie, and Robert Tibshirani. 2001. The elements of statistical learning. Vol. 1. Springer series in statistics New York.

[8] Greenplum. 2019. Greenplum website. greenplum.org

[9] H2O. 2009. H2O.ai. www.h2o.ai

[10] Joseph M. Hellerstein, Christoper Ré, Florian Schoppmann, Daisy Zhe Wang, Eugene Fratkin, Aleksander Gorajek, Kee Siong Ng, Caleb Welton, Xixuan Feng, Kun Li, and Arun Kumar. 2012. The MADlib Analytics Library: Or MAD Skills, the SQL. Proc. VLDB Endow. 5, 12 (Aug. 2012), 1700-1711. https://doi.org/10.14778/2367502.2367510

[11] Joab Jackson. 2018. Google Adds Machine Learning Modeling to BigQuery. thenewstack.io/google-adds-machine-learning-modelingto-bigquery/ 
[12] Dimitrije Jankov, Shangyu Luo, Binhang Yuan, Zhuhua Cai, Jia Zou, Chris Jermaine, and Zekai J. Gao. 2019. Declarative Recursive Computation on an RDBMS: Or, Why You Should Use a Database for Distributed Machine Learning. Proc. VLDB Endow. 12, 7 (March 2019), 822-835. https://doi.org/10.14778/3317315.3317323

[13] Kaggle. 2019. Diamonds-Kaggle Dataset. www.kaggle.com/ shivam2503/diamonds

[14] Andrew Lamb, Matt Fuller, Ramakrishna Varadarajan, Nga Tran, Ben Vandiver, Lyric Doshi, and Chuck Bear. 2012. The vertica analytic database: C-store 7 years later. Proceedings of the VLDB Endowment 5, 12 (2012), 1790-1801.

[15] Jeff LeFevre, Rui Liu, Cornelio Inigo, Lupita Paz, Edward Ma, Malu Castellanos, and Meichun Hsu. 2016. Building the Enterprise Fabric for Big Data with Vertica and Spark Integration. In SIGMOD. ACM, 63-75.

[16] MADlib. 2019. MADlib website. madlib.apache.org/

[17] Ryan Marcus, Parimarjan Negi, Hongzi Mao, Chi Zhang, Mohammad Alizadeh, Tim Kraska, Olga Papaemmanouil, and Nesime Tatbul. 2019. Neo: A Learned Query Optimizer. Proc. VLDB Endow. 12, 11 (July 2019), 1705-1718. https://doi.org/10.14778/3342263.3342644

[18] Xiangrui Meng, Joseph Bradley, Burak Yavuz, Evan Sparks, Shivaram Venkataraman, Davies Liu, Jeremy Freeman, DB Tsai, Manish Amde, Sean Owen, et al. 2016. MLlib: Machine learning in Apache Spark. The Journal of Machine Learning Research 17, 1 (2016), 1235-1241.

[19] Microsoft. 2009. Microsoft SQL MLS. docs.microsoft.com/enus/sql/advanced-analytics/what-is-sql-server-machinelearning?view=sql-server -2017

[20] Oracle. 2019. Oracle Advanced Analytics. www.oracle.com/ technetwork/database/options/advanced-analytics/overview/index. html

[21] Biswanath Panda, Joshua S. Herbach, Sugato Basu, and Roberto J. Bayardo. 2009. PLANET: Massively Parallel Learning of Tree Ensembles with MapReduce. Proc. VLDB Endow. 2, 2 (Aug. 2009), 1426-1437. https://doi.org/10.14778/1687553.1687569

[22] Neoklis Polyzotis, Sudip Roy, Steven Euijong Whang, and Martin Zinkevich. 2018. Data lifecycle challenges in production machine learning: A survey. ACM SIGMOD Record 47, 2 (2018), 17-28.

[23] PostgreSQL. 2019. PostgreSQL website. www.postgresql.org

[24] Christopher Ré, Divy Agrawal, Magdalena Balazinska, Michael Cafarella, Michael Jordan, Tim Kraska, and Raghu Ramakrishnan. 2015. Machine learning and databases: The sound of things to come or a cacophony of hype?. In SIGMOD. ACM, 283-284.

[25] Seyed H. Roosta. 1999. Parallel Processing and Parallel Algorithms: Theory and Computation (1st ed.). Springer-Verlag, Berlin, Heidelberg.

[26] Vinay Sridhar, Sriram Subramanian, Dulcardo Arteaga, Swaminathan Sundararaman, Drew Roselli, and Nisha Talagala. 2018. Model governance: Reducing the anarchy of production ML. In USENIX. 351-358.

[27] Mike Stonebraker, Daniel J Abadi, Adam Batkin, Xuedong Chen, Mitch Cherniack, Miguel Ferreira, Edmond Lau, Amerson Lin, Sam Madden, Elizabeth O'Neil, et al. 2005. C-store: a column-oriented DBMS. In VLDB. VLDB Endowment, 553-564.

[28] Manasi Vartak and Samuel Madden. 2018. MODELDB: Opportunities and Challenges in Managing Machine Learning Models. Data Engineering (2018), 16.

[29] Vertica. 2019. Vertica-SDK. www.vertica.com/docs/9.3.x/HTML/ Content/Home.htm

[30] Hadley Wickham, Romain Francois, L Henry, and K Müller. 2017. dplyr: A Grammar of Data Manipulation. R package version 0.7.4. 